\title{
CRITICAL GROUPS COMPUTATIONS ON A CLASS OF SOBOLEV BANACH SPACES VIA MORSE INDEX
}

\author{
Silvia CINGOLANI ${ }^{*, 1}$, Giuseppina VANNELLA ${ }^{1}$
}

Dipartimento Interuniversitario di Matematica, Politecnico di Bari, via E. Orabona 4, 70125 Bari, Italy

Received 22 April 2001, revised 26 October 2001

ABSTRACT. - In this paper we deal with critical groups estimates for a functional $f: W_{0}^{1, p}(\Omega)$ $\rightarrow \mathbb{R}(p>2), \Omega$ bounded domain of $\mathbb{R}^{N}$, defined by setting

$$
f(u)=\frac{1}{p} \int_{\Omega}|\nabla u|^{p} d x+\frac{1}{2} \int_{\Omega}|\nabla u|^{2} d x+\int_{\Omega} G(u) d x
$$

where $G(t)=\int_{0}^{t} g(s) d s$ and $g$ is a smooth real function on $\mathbb{R}$, growing subcritically. We remark that the second derivative of $f$ in each critical point $u$ is not a Fredholm operator from $W_{0}^{1, p}(\Omega)$ to its dual space, so that the generalized Morse splitting lemma does not work. In spite of the lack of an Hilbert structure, we compute the critical groups of $f$ in $u$ via its Morse index. (c) 2003 L'Association Publications de l'Institut Henri Poincaré. Published by Elsevier B.V. All rights reserved

Keywords: Morse theory; Critical groups estimate; p-Laplacian

RÉSUMÉ. - Dans cet article, nous estimons les groupes critiques pour une fonctionnelle : $f: W_{0}^{1, p}(\Omega) \rightarrow \mathbb{R}(p>2), \Omega$ ensemble borné de $\mathbb{R}^{N}$, définie par

$$
f(u)=\frac{1}{p} \int_{\Omega}|\nabla u|^{p} d x+\frac{1}{2} \int_{\Omega}|\nabla u|^{2} d x+\int_{\Omega} G(u) d x
$$

où $G(t)=\int_{0}^{t} g(s) d s$ et $g$ est une fonction réelle et régulière sur $\mathbb{R}$, avec une croissance souscritique. On observe que la dérive seconde de $f$ en chaque point $u$ n'est pas un opérateur de Fredholm entre $W_{0}^{1, p}(\Omega)$ est son espace dual et la généralisation du Lemme de Morse ne peut être appliquée. Bien que la structure d'espace d'Hilbert n'existe pas, on est capable de calculer les groupes critiques de $f$ en $u$ grâce à l'index de Morse.

(c) 2003 L'Association Publications de l'Institut Henri Poincaré. Published by Elsevier B.V. All rights reserved

\footnotetext{
* Corresponding author.

E-mail addresses: cingolan@dm.uniba.it (S. Cingolani), vannella@dm.uniba.it (G. Vannella).

${ }^{1}$ Supported by MURST Research funds.
} 


\section{Introduction and statement of the results}

Since its infinite dimensional generalizations, carried out independently by Palais [17] and Smale [18], Morse theory has been widely used in the study of multiplicity of solutions for semilinear elliptic boundary value problems, arising in the calculus of variations. It is standard that the solutions of such a differential problem can be seen as critical points of a suitable smooth energy functional $f$, defined on a Hilbert space $H$. The basic idea of Morse theory is that the number of solutions of the differential problem can be estimated by investigating the variations of the topological structures of the level sets of $f$. Therefore it becomes crucial to describe locally the behavior of the energy functional near its critical points. A way to investigate such a behavior is to evaluate the critical groups at the isolated critical points (see Definition 2.1 in Section 2 below).

We recall here that a critical point $u$ of $f$ is classically said to be nondegenerate if the second order differential $f^{\prime \prime}(u): H \rightarrow H^{*}$ is an isomorphism. Such definition ensures that any nondegenerate critical point is isolated and the Morse splitting lemma holds. As a consequence the local behavior of the functional near any nondegenerate critical point is quite clear and computing the critical groups of a nondegenerate critical point is possible via its Morse index, namely the supremum of the dimensions of the subspaces on which $f^{\prime \prime}(u)$ is negative definite (see Theorem 2.4). Successively, these ideas are generalized by Gromoll and Meyer for computing the critical groups of an isolated critical point $u$, possibly degenerate, with finite Morse index, in the case $f^{\prime \prime}(u)$ is a Fredholm operator (see Theorem 2.5). The generalized Morse lemma is a basic tool for the effective computation of the critical groups and the theory of Fredholm operators provides a natural setting for this lemma. Moreover we emphasize that such critical groups estimates seem to require an Hilbert space structure.

However, in the study of nonlinear partial differential equations, sometimes we are led to work on Banach spaces rather than Hilbert spaces. This is the case when we deal with quasilinear elliptic equations involving $p$-laplacian $(p>2)$. Then, it seems rather natural to ask whether it is possible to extend Morse theory to Banach spaces and describe locally the behavior of the energy functional associated to the quasilinear elliptic problems through the estimates of its critical groups.

In order to give an answer to this question, some conceptual difficulties arise. A main difficulty is the lack of a proper definition of nondegenerate critical point. Indeed, if $X$ is a Banach space and $f: X \rightarrow \mathbb{R}$ is a $C^{2}$ functional, any critical point of $f$ is degenerate, according to the definition given in a Hilbert space, if $X$ is not isomorphic to the dual space $X^{*}$. Moreover, it is possible to prove that the existence of a nondegenerate critical point $u \in X$ of $f$ having finite Morse index, which is the most interesting case in Morse theory, implies the existence on $X$ of an equivalent Hilbert structure (see [16]). Furthermore, if $X$ is a Banach space, which is not isomorphic to its dual, a second difficulty arises. In such a case, generalized splitting Morse lemmas fail (see, for example, Theorem 2.5), as $f^{\prime \prime}(u)$ cannot be a Fredholm operator.

As the definition of nondegenerate critical point given in a Hilbert space does not work in a Banach space, in literature some authors have introduced different weaker nondegeneracy conditions for the critical points of functionals defined on a Banach space 
(see e.g., $[4,22,23])$. However these nondegeneracy conditions seem to be rather involved and in general not easy to be verified.

In this paper we aim to estimate the critical groups of some functionals associated to a class of quasilinear elliptic problems, involving $p$-laplacian. Hence we shall focus on a class of Banach spaces which are the natural variational setting for several quasilinear elliptic problems. Precisely, we shall work on the Sobolev space $W_{0}^{1, p}(\Omega)$, where $2<p<\infty$ and $\Omega$ is a bounded domain of $\mathbb{R}^{N}(N \geqslant 1)$, with sufficiently regular boundary $\partial \Omega$. We shall consider the functional $f: W_{0}^{1, p}(\Omega) \rightarrow \mathbb{R}$ defined by setting

$$
f(u)=\frac{1}{p} \int_{\Omega}|\nabla u|^{p} d x+\frac{1}{2} \int_{\Omega}|\nabla u|^{2} d x+\int_{\Omega} G(u) d x
$$

where $G(t)=\int_{0}^{t} g(s) d s$ and $g \in C^{1}(\mathbb{R}, \mathbb{R})$ satisfies the following assumption:

(g) $\left|g^{\prime}(t)\right| \leqslant c_{1}|t|^{q}+c_{2}$ with $c_{1}, c_{2}$ positive constants and $0 \leqslant q<p^{*}-2$ if $N>p$, while $q$ is any positive number, if $N=p$.

Otherwise, if $N<p$, no restrictive assumption on the growth of $g$ is required.

Clearly, critical points of the functional $f$ in $W_{0}^{1, p}(\Omega)$ correspond to weak solutions of the quasilinear elliptic problem

$$
\begin{cases}-\Delta_{p} u-\Delta u+g(u)=0 & \text { in } \Omega, \\ u=0 & \text { on } \partial \Omega,\end{cases}
$$

where $\Delta_{p} u=\operatorname{div}\left(|\nabla u|^{p-2} \nabla u\right), p>2$, arising in the mathematical description of propagation phenomena of solitary waves (cf. [2,3]).

We point out that, as $W_{0}^{1, p}(\Omega)$ is not isomorphic to the dual space $W^{-1, p^{\prime}}(\Omega)$, where $1 / p+1 / p^{\prime}=1$, any critical point $u$ of $f$ is degenerate, in the sense already given for Hilbert spaces. Furthermore, as $f^{\prime \prime}(u)$ is not a Fredholm operator, we cannot apply the generalized splitting Morse lemma in order to describe the behavior of $f$ near the critical points.

In spite of these difficulties, we are able to obtain critical groups estimates for functional $f$ in $u$, in the spirit of the generalized Morse lemma.

Before stating the main results, let us denote by $m(f, u)$ the Morse index of $f$ in $u$ and by $m^{*}(f, u)$ the sum of $m(f, u)$ and the dimension of the kernel of $f^{\prime \prime}(u)$ in $W_{0}^{1, p}(\Omega)$. Moreover let $C_{q}(f, u)$ be the $q$ th critical group of $f$ in $u$ with respect to a field $\mathbb{K}$, which is well defined according to Definition 2.1.

THEOREM 1.1. - Let $u$ be an isolated critical point of the functional (1.1) such that $f^{\prime \prime}(u)$ is injective. Then $m(f, u)$ is finite and

$$
\begin{aligned}
& C_{q}(f, u) \cong \mathbb{K}, \quad \text { if } q=m(f, u), \\
& C_{q}(f, u)=\{0\}, \quad \text { if } q \neq m(f, u) .
\end{aligned}
$$

This theorem extends a classical result in Hilbert spaces for nondegenerate critical points (cf. Theorem 2.4), showing that the critical groups of $f$ in $u$ depend only upon its Morse index. It is interesting to observe that the usual nondegeneracy condition, 
namely $f^{\prime \prime}(u)$ is an isomorphism, can be weakened by requiring only the injectivity. As mentioned by Uhlenbeck in [23], in an unpublished article Smale conjectured that the nondegeneracy condition $f^{\prime \prime}(u)$ has no kernel could be sufficient to develop Morse theory. Theorem 1.1 shows that the Smale's conjecture is true for the functional (1.1). So this suggests a new definition of nondegenerate critical point for the class of functionals (1.1), i.e.,

$u$ is a nondegenerate critical point of $f$ if $f^{\prime \prime}(u): W_{0}^{1, p}(\Omega) \rightarrow W_{0}^{-1, p^{\prime}}(\Omega)$ is injective.

In the case in which $f^{\prime \prime}(u)$ is not injective, we shall prove that the number of nontrivial critical groups of $f$ in $u$ is finite. Precisely, we state the following result.

THEOREM 1.2. - Let $u$ be an isolated critical point of the functional (1.1). Then $m(f, u)$ and $m^{*}(f, u)$ are finite and

$$
C_{q}(f, u)=\{0\}
$$

for any $q \leqslant m(f, u)-1$ and $q \geqslant m^{*}(f, u)+1$.

In the proof of Theorems 1.1 and 1.2, the computations of the critical groups of $f$ in the isolated critical point $u$ is reduced to a finite dimensional problem. The main used tool is the space $H_{u}=W_{0}^{1,2}(\Omega)$ equipped by an auxiliary Hilbert structure, which depends on the critical point $u$, being suggested by $f^{\prime \prime}(u)$ itself. In such way $W_{0}^{1, p}(\Omega)$ is continuously imbedded on $H_{u}$ and $f^{\prime \prime}(u)$ can be extended to a Fredholm operator $L_{u}$ from $W_{0}^{1,2}(\Omega)$ to its dual space, so that the dimension of the kernel of $L_{u}$ in $W_{0}^{1,2}(\Omega)$ is finite. Furthermore we can consider the natural splitting

$$
W_{0}^{1,2}(\Omega)=H^{-} \oplus H^{0} \oplus H^{+}
$$

where $H^{-}, H^{0}, H^{+}$are, respectively, the negative, null, and positive spaces, according to the spectral decomposition of $L_{u}$ in $L^{2}(\Omega)$.

By regularity arguments, we can also split the Banach space $W_{0}^{1, p}(\Omega)$, yielding that $m(f, u)$ and $m^{*}(f, u)$ are finite.

In the case $N<p$, we also enhance a kind of uniform convexity of $f$ near $u$ along the positive space $H^{+} \cap W_{0}^{1, p}(\Omega)$, with respect to a weaker norm than the usual one of $W_{0}^{1, p}(\Omega)$. This is enough to obtain a suitable reduction to finite dimension.

In the case $N \geqslant p$ we lose the "uniform weak convexity" of $f$ near $u$, but we are able to prove a sort of local convexity in the bounded sets of $L^{\infty}(\Omega)$ along the direction of $H^{+} \cap W_{0}^{1, p}(\Omega)$. In this case the reduction to a finite dimensional problem is more complicated and require some arguments strictly related to the Second Deformation Lemma (cf. [5,6]).

For reader's convenience, in many points of this work, we prefer to distinguish the two cases $N<p$ and $N \geqslant p$.

We quote that in a recent paper [14], a finiteness result on the nontrivial critical groups for continuous functionals, defined on a Hilbert space, is obtained by means of a reduction to a finite dimensional problem. Concerning critical groups estimates we also mention the papers by $[6,8]$. 
In a forthcoming paper, the critical groups estimates, obtained in Theorems 1.1 and 1.2, will be applied to get a multiplicity result for a quasilinear elliptic problem of the type

$$
\begin{cases}-\varepsilon^{p} \Delta_{p} u-\varepsilon^{2} \Delta u+g(u)=0 & \text { in } \Omega, \\ u=0 & \text { on } \partial \Omega\end{cases}
$$

for small $\varepsilon>0$. Such a quasilinear problem arises when looking for static solutions for a model equation, which admits soliton-like solutions, in the spirit of [2].

The results of the present paper have been announced in [7].

\section{Critical groups in Morse Theory}

In this section we recall some notions and results in Morse Theory.

Let $X$ be a Banach space and $f$ be a $C^{2}$ real function on $X$. For any $a \in \mathbb{R}$, we will use the following notations:

$$
\begin{aligned}
& f^{a}=\{x \in X: f(x) \leqslant a\}, \\
& K=\left\{x \in X: f^{\prime}(x)=0\right\} .
\end{aligned}
$$

DEFINITION 2.1. - Let $\mathbb{K}$ be a field. Let $u$ be an isolated critical point of $f$, and let $c=f(u)$. We call

$$
C_{q}(f, u)=H^{q}\left(f^{c}, f^{c} \backslash\{u\}\right)
$$

the qth critical group of $f$ at $u, q=0,1,2, \ldots$, where $H^{q}(A, B)$ stands for the qth Alexander-Spanier cohomology group of the pair $(A, B)$ with coefficients in $\mathbb{K}$ (cf. [19]).

Remark 2.2. - By excision property, we have also that, if $U$ is a neighborhood of $u$, then

$$
C_{q}(f, u) \cong H^{q}\left(f^{c} \cap U,\left(f^{c} \backslash\{u\}\right) \cap U\right) .
$$

Now let us fix $u \in X$ a critical point of $f$. We recall the following definition.

DEFINITION 2.3. - The Morse index of $f$ in $u$ is the supremum of the dimensions of the subspaces of $X$ on which $f^{\prime \prime}(u)$ is negative definite. It is denoted by $m(f, u)$. Moreover, the large Morse index of $f$ in $u$ is the sum of $m(f, u)$ and the dimension of the kernel of $f^{\prime \prime}(u)$. It is denoted by $m^{*}(f, u)$.

Now we consider a Hilbert space $H$ and a $C^{2}$ functional $f$ on $H$. As mentioned in the introduction, a critical point $u \in H$ of $f$ is said nondegenerate, if $f^{\prime \prime}(u)$ has a bounded inverse. As showed in the following result, it is possible to compute the critical groups of a nondegenerate critical point via its Morse index, using the Morse Lemma (see, for example, [5,15]).

THEOREM 2.4.-Suppose $H$ a Hilbert space and $f \in C^{2}(H, \mathbb{R})$. Let $u$ be a nondegenerate critical point of $f$ with Morse index $m$. Then

$$
C_{q}(f, u) \cong \mathbb{K} \quad \text { if } q=m, \quad C_{q}(f, u)=\{0\} \quad \text { if } q \neq m .
$$


Nevertheless, if $m=+\infty$, we always have

$$
C_{q}(f, u)=\{0\} .
$$

These ideas are extended by Gromoll and Meyer for computing the critical groups of isolated critical points, possibly degenerate, of functional defined on a Hilbert space. For completeness, we recall the generalized Morse lemma (see, for example, [5]).

THEOREM 2.5. - Suppose $H$ a Hilbert space and $f \in C^{2}(H, \mathbb{R})$. Let $u$ be an isolated critical point of $f$ with Morse index $m$ and large Morse index $m^{*}$. Suppose that $f^{\prime \prime}(u)$ is a Fredholm operator and let $N$ be the kernel of $f^{\prime \prime}(u)$. If $u$ is a local minimum of $\tilde{f}=f_{\mid N}$, then

$$
C_{q}(f, u) \cong \delta_{q, m} \mathbb{K} .
$$

Moreover if $u$ is a local maximum of $\tilde{f}=f_{\mid N}$, then

$$
C_{q}(f, u) \cong \delta_{q, m^{*}} \mathbb{K} \text {. }
$$

If $u$ is neither a local maximum nor a local minimum of $\tilde{f}$, then

$$
C_{q}(f, u)=\{0\} \quad \text { if } q \leqslant m, \quad C_{q}(f, u)=\{0\} \quad \text { if } q \geqslant m^{*} .
$$

Finally we recall the following result proved by Lancelotti [14] concerning critical groups estimates.

THEOREM 2.6. - Let $X$ be a Banach space, $f: X \rightarrow \mathbb{R}$ a continuous function and $V$ a subspace of $X$ of finite dimension $m$. We assume that:

(i) for every $u \in X$, the function $f$ is of class $C^{2}$ on $u+V$ and for every $v \in V$ the functions $u \mapsto\left\langle f^{\prime}(u) v\right\rangle$ and $u \mapsto\left\langle f^{\prime \prime}(u) v, v\right\rangle$ are continuous on $X$;

(ii) $\left\langle f^{\prime \prime}(u) v, v\right\rangle<0$ for every $v \in V \backslash\{0\}$.

Then we have $C_{q}(f, u)=\{0\}$ for every $q \leqslant m-1$.

\section{An auxiliary Hilbert space}

In what follows, we denote by $(\cdot \mid \cdot)$ the scalar product in $\mathbb{R}^{N}$, by $\|\cdot\|_{\infty}$ and $\|\cdot\|$ the usual norms in $L^{\infty}(\Omega)$ and $W_{0}^{1, p}(\Omega)$, respectively. Here $2<p<+\infty, \Omega$ is a bounded domain in $\mathbb{R}^{N}$ with sufficiently regular boundary. Let us denote $B_{r}(u)=$ $\left\{v \in W_{0}^{1, p}(\Omega):\|v-u\|<r\right\}$, where $u \in W_{0}^{1, p}(\Omega)$ and $r>0$. Moreover we denote by $\langle\cdot, \cdot\rangle: W^{-1, p^{\prime}}(\Omega) \times W_{0}^{1, p}(\Omega) \rightarrow \mathbb{R}$ the duality pairing.

Let us consider the functional $f: W_{0}^{1, p}(\Omega) \rightarrow \mathbb{R}$ defined in (1.1) by setting

$$
f(u)=\frac{1}{p} \int_{\Omega}|\nabla u|^{p} d x+\frac{1}{2} \int_{\Omega}|\nabla u|^{2} d x+\int_{\Omega} G(u) d x
$$

where $G(t)=\int_{0}^{t} g(s) d s$ and $g \in C^{1}(\mathbb{R}, \mathbb{R})$ satisfying the assumption $(g)$ in the introduction. Standard arguments prove that $f$ is a $C^{2}$ functional on $W_{0}^{1, p}(\Omega)$. 
Let us fix an isolated critical point $u \in W_{0}^{1, p}(\Omega)$ of $f$ and set $c=f(u)$. It is easy to prove that the second order differential of $f$ in $u$ is given by

$$
\begin{aligned}
\left\langle f^{\prime \prime}(u) v, w\right\rangle= & \int_{\Omega}\left(1+|\nabla u|^{p-2}\right)(\nabla v \mid \nabla w) d x \\
& +\int_{\Omega}(p-2)|\nabla u|^{p-4}(\nabla u \mid \nabla v)(\nabla u \mid \nabla w) d x+\int_{\Omega} g^{\prime}(u) v w d x
\end{aligned}
$$

for any $v, w \in W_{0}^{1, p}(\Omega)$.

As mentioned in the introduction, since $W_{0}^{1, p}(\Omega)(p>2)$ is not isomorphic to its dual space, the operator $f^{\prime \prime}(u)$ is not a Fredholm operator, thus any generalized splitting Morse lemma fails. To overcome this difficulty, we introduce a Hilbert space, depending on the critical point $u$, in which $W_{0}^{1, p}(\Omega)$ can be embedded, so that a natural splitting can be obtained.

To this aim we need to prove some regularity result on the critical point $u$. We point out that in the case $N<p$, the Sobolev space $W_{0}^{1, p}(\Omega)$ is continuously embedded in $L^{\infty}(\Omega)$, and thus $u \in L^{\infty}(\Omega)$. Conversely, in the case $N \geqslant p$, we can also derive the $L^{\infty}$-boundedness of the critical point $u$, arguing as in Lemma 1.4 in [1].

LEMMA 3.1. - Let $u \in W_{0}^{1, p}(\Omega)$ be a critical point of $f$. Then $u \in L^{\infty}(\Omega)$.

Proof. - The case $N<p$ is trivial, as $W_{0}^{1, p}(\Omega)$ is continuously embedded in $L^{\infty}(\Omega)$. Now we focus on the case $N \geqslant p$. For $j \in \mathbb{N}$ let us consider the real functions $\chi_{j}$ defined by $\chi_{j}(z)=z+j$ if $z<-j, \chi_{j}(z)=0$ if $-j \leqslant z \leqslant j, \chi_{j}(z)=z-j$ if $j<z$.

Taking $v=\chi_{j}(u) \in W_{0}^{1, p}(\Omega)$ as test function in the equation $f^{\prime}(u)=0$, we deduce

$$
\int_{\Omega}\left|\nabla \chi_{j}(u)\right|^{p} d x+\int_{\Omega}\left|\nabla \chi_{j}(u)\right|^{2} d x+\int_{\Omega} g(u) \chi_{j}(u) d x=0 .
$$

Consequently, taking into account that $\left|\chi_{j}(u)\right| \leqslant|u|$ and by assumption $(g)$, we obtain

$$
\int_{\Omega_{j}}|\nabla u|^{p} d x \leqslant c_{1} \int_{\Omega_{j}}|u|^{q+2} d x+c_{2} \int_{\Omega_{j}}|u| d x
$$

where $\Omega_{j}=\{x \in \Omega:|u(x)|>j\}$ and $c_{1}, c_{2}$ are suitable positive constants. Applying inequality (3.4), Chapter 5 in [13], we conclude that $u \in L^{\infty}(\Omega)$.

Furthermore, since $u \in W_{0}^{1, p}(\Omega) \cap L^{\infty}(\Omega)$, we can infer that $u \in C^{1}(\bar{\Omega})$. The local result can be found in $[9,20]$. The proof that $u$ has locally Holder continuous derivatives at the boundary is given in [21] (see also [10]).

At this point, let $b(x)=|\nabla u(x)|^{(p-4) / 2} \nabla u(x) \in L^{\infty}(\Omega)$. Let $H_{b}$ be the closure of $C_{0}^{\infty}(\Omega)$ under the scalar product

$$
(v, w)_{b}=\int_{\Omega}\left(1+|b|^{2}\right)(\nabla v \mid \nabla w) d x+(p-2)(b \mid \nabla v)(b \mid \nabla w) d x
$$


Let us define $\|\cdot\|_{b}$ the norm induced by $(\cdot, \cdot)_{b}$. Clearly $\|\cdot\|_{b}$ is equivalent to the usual norm of $W_{0}^{1,2}(\Omega)$, denoted by $\|\cdot\|_{W_{0}^{1,2}}$, and so $H_{b}$ is isomorphic to $W_{0}^{1,2}(\Omega)$. Thus $W_{0}^{1, p}(\Omega) \subset H_{b}$ continuously.

Now let us define $L_{b}: H_{b} \rightarrow H_{b}^{*}$ by setting

$$
\left\langle L_{b} v, w\right\rangle=(v, w)_{b}+\langle K v, w\rangle
$$

where $\langle K v, w\rangle=\int_{\Omega} g^{\prime}(u) v w d x$ for any $v, w \in H_{b}$.

LEMMA 3.2. $-L_{b}$ is a compact perturbation of the Riesz isomorphism from $H_{b}$ to $H_{b}^{*}$. In particular, $L_{b}$ is a Fredholm operator with index zero.

Proof. - In order to prove the assert it is sufficient to show that $K$ is a compact operator from $H_{b}$ to $H_{b}^{*}$. Let $\left\{v_{n}\right\}$ be a bounded sequence in $H_{b}$. Then there exists $v \in H_{b}$ such that $\left\{v_{n}\right\}$ converges, up to a subsequence, to $v$ weakly in $H_{b}$ and strongly in $L^{2}(\Omega)$. By Lemma 3.1 and the continuity of $g^{\prime}$, there is a constant $c>0$ such that, for any $w \in H_{b}$, $\|w\|_{b}=1$ we have

$$
\left|\int_{\Omega} g^{\prime}(u)\left(v_{n}-v\right) w d x\right| \leqslant \int_{\Omega}\left|g^{\prime}(u)\right|\left|v_{n}-v\right||w| d x \leqslant c\left(\int_{\Omega}\left|v_{n}-v\right|^{2} d x\right)^{1 / 2}
$$

which tends to zero as $n \rightarrow+\infty$, uniformly with respect to $w$. This implies that $K$ is a compact operator.

Now let us denote by $m\left(L_{b}\right)$ the maximal dimension of a subspace of $H_{b}$ on which $L_{b}$ is negative definite. Obviously $m(f, u) \leqslant m\left(L_{b}\right)$. Furthermore let us denote by $m^{*}\left(L_{b}\right)$ the sum of $m\left(L_{b}\right)$ and the dimension of the kernel of $L_{b}$. By Lemma 3.2 we conclude that $m\left(L_{b}\right)$ and $m^{*}\left(L_{b}\right)$ are finite.

Since $L_{b}$ is a Fredholm operator in $H_{b}$, we can consider the natural splitting

$$
H_{b}=H^{-} \oplus H^{0} \oplus H^{+}
$$

where $H^{-}, H^{0}, H^{+}$are, respectively, the negative, null, and positive spaces, according to the spectral decomposition of $L_{b}$ in $L^{2}(\Omega)$.

Furthermore, it is obvious that there exists $c>0$ such that

$$
\left\langle L_{b} v, v\right\rangle_{b}+c \int_{\Omega} v^{2} d x \geqslant\|v\|_{b}^{2} \quad \forall v \in H_{b}
$$

Therefore, one can easily show that

$$
\exists \mu>0 \quad \text { s.t. } \quad\left\langle L_{b} v, v\right\rangle \geqslant \mu\|v\|_{b}^{2} \quad \forall v \in H^{+}
$$

and $m\left(L_{b}\right), m^{*}\left(L_{b}\right)$ are, respectively, the dimensions of $H^{-}$and $H^{-} \oplus H^{0}$.

Since $u \in C^{1}(\bar{\Omega})$, we can deduce from standard regularity theory (see Theorems 8.15 , 8.24 and 8.29 in [11]) that

$$
H^{-} \oplus H^{0} \subset W_{0}^{1, p}(\Omega) \cap L^{\infty}(\Omega) .
$$


Consequently, denoted by $W=H^{+} \cap W_{0}^{1, p}(\Omega)$ and $V=H^{-} \oplus H^{0}$, we get the splitting

$$
W_{0}^{1, p}(\Omega)=V \oplus W
$$

and, by (3.1)

$$
\left\langle f^{\prime \prime}(u) v, v\right\rangle \geqslant \mu\|v\|_{b}^{2} \quad \forall v \in W
$$

so that

$$
m\left(L_{b}\right)=m(f, u), \quad m^{*}\left(L_{b}\right)=m^{*}(f, u) .
$$

\section{Some useful lemmas}

We start to recall an abstract result, contained in [12], which gives a description of integrands generating integral functionals which are sequentially lower semicontinuous with respect to mixed strong-weak convergence.

THEOREM 4.1. - Let $\left.\left.\Phi: L^{p}\left(\Omega, \mathbb{R}^{k}\right) \times L^{q}\left(\Omega, \mathbb{R}^{m}\right) \rightarrow\right]-\infty,+\infty\right]$ be a functional of the form

$$
\Phi(u, v)=\int_{\Omega} \phi(x, u, v) d x
$$

where $\phi(x, u, v)$ is a nonnegative, continuous function and $\phi(x, u, \cdot)$ is convex. Then $\Phi$ is lower semicontinuous with respect to the strong convergence of the component $u$ in $L^{p}$ and with respect to the weak convergence of the component $v$ in $L^{q}$.

The above abstract theorem is useful in order to prove some lemmas concerning, in some sense, the uniform convexity of $f$ near the critical point $u$ in the direction of $W$. We need to distinguish the two cases $N<p$ and $N \geqslant p$. We start to prove the following lemma.

Lemma 4.2. - Let $N<p$. There exist $r_{0}>0$ and $C>0$ such that for any $z \in$ $W_{0}^{1, p}(\Omega),\|z-u\|<r_{0}$, we have

$$
\left\langle f^{\prime \prime}(z) v, v\right\rangle \geqslant C\|v\|_{b}^{2}
$$

for any $v \in W$.

Proof. - By contradiction, we assume that there exist two sequences $z_{n} \in W_{0}^{1, p}(\Omega)$ and $v_{n} \in W \backslash\{0\},\left\|v_{n}\right\|_{W_{0}^{1,2}}=1$, such that $\left\|z_{n}-u\right\| \rightarrow 0$ and

$$
\liminf _{n \rightarrow \infty}\left\langle f^{\prime \prime}\left(z_{n}\right) v_{n}, v_{n}\right\rangle \leqslant 0 .
$$

Since $\left\{v_{n}\right\}$ is bounded in $H_{b}$, there exists $v \in W$ such that $v_{n}$ converges to $v$ weakly in $H_{b}$ and strongly in $L^{2}(\Omega)$, up to subsequences. Firstly we prove that $v \neq 0$. By contradiction we assume that $v=0$. For simplicity, we denote $h(\xi)=\frac{1}{p}|\xi|^{p}, \xi \in \mathbb{R}^{N}$. We notice that 


$$
\begin{aligned}
\left\langle f^{\prime \prime}\left(z_{n}\right) v_{n}, v_{n}\right\rangle & =\int_{\Omega}\left|\nabla v_{n}\right|^{2} d x+\int_{\Omega}\left(h^{\prime \prime}\left(\nabla z_{n}\right) \nabla v_{n} \mid \nabla v_{n}\right) d x+\int_{\Omega} g^{\prime}\left(z_{n}\right) v_{n}^{2} d x \\
& \geqslant 1+\int_{\Omega} g^{\prime}\left(z_{n}\right) v_{n}^{2} d x .
\end{aligned}
$$

By letting $n \rightarrow+\infty$, we get $\int_{\Omega} g^{\prime}\left(z_{n}\right) v_{n}^{2} d x \rightarrow 0$ and by (4.2) and (4.3) we obtain a contradiction.

Moreover, since $\left(h^{\prime \prime}(\nabla u) \nabla v \mid \nabla v\right)$ is convex with respect to $\nabla v$, by Theorem 4.1, we infer

$$
\int_{\Omega}\left(h^{\prime \prime}(\nabla u) \nabla v \mid \nabla v\right) d x \leqslant \liminf _{n \rightarrow \infty} \int_{\Omega}\left(h^{\prime \prime}\left(\nabla z_{n}\right) \nabla v_{n} \mid \nabla v_{n}\right) d x .
$$

Then (4.2) and (4.4) imply

$$
0 \geqslant \liminf _{n \rightarrow \infty}\left\langle f^{\prime \prime}\left(z_{n}\right) v_{n}, v_{n}\right\rangle \geqslant\left\langle L_{b} v, v\right\rangle \geqslant \mu\|v\|_{b}^{2},
$$

which is a contradiction.

We remark that if $N \geqslant p$ we are not able to prove Lemma 4.2. Nevertheless a weaker result can be obtained concerning, in some sense, the uniform convexity of $f$ near $u$ in the bounded set of $L^{\infty}(\Omega)$, along the direction of $W$ (see Lemma 4.4). We firstly need the following regularity result.

LEMMA 4.3. - Let $a>0$. If $z \in B_{a}(u) \cap W_{0}^{1, p}(\Omega)$ is a solution of

$$
\int_{\Omega}(\nabla z \mid \nabla w) d x+\int_{\Omega}|\nabla z|^{p-2}(\nabla z \mid \nabla w) d x+\int_{\Omega} g(z) w d x=0
$$

for any $w \in W$, then $z \in L^{\infty}(\Omega)$. Moreover there exists $K>0$ such that $\|z\|_{\infty} \leqslant K$, with $K$ depending on $a$.

Proof. - Let $\left\{e_{1}, \ldots, e_{m^{*}}\right\}$ be an $L^{2}$-orthonormal basis in $V$, where $m^{*}=m^{*}(f, u)$. For any $v \in W_{0}^{1, p}(\Omega)$, we can choose $v-\sum_{i=1}^{m^{*}}\left(\int_{\Omega} e_{i} v d x\right) e_{i} \in W$ as test function in (4.5). Therefore, we get

$$
\begin{aligned}
\int_{\Omega} & (\nabla z \mid \nabla v) d x+\int_{\Omega}|\nabla z|^{p-2}(\nabla z \mid \nabla v) d x+\int_{\Omega} g(z) v d x \\
= & \sum_{i=1}^{m^{*}}\left(\int_{\Omega} e_{i} v d x\right)\left(\int_{\Omega}\left(\nabla z \mid \nabla e_{i}\right)+|\nabla z|^{p-2}\left(\nabla z \mid \nabla e_{i}\right)+g(z) e_{i} d x\right) .
\end{aligned}
$$

Let us define

$$
r(x)=\sum_{i=1}^{m^{*}}\left(\int_{\Omega}\left(\nabla z \mid \nabla e_{i}\right)+|\nabla z|^{p-2}\left(\nabla z \mid \nabla e_{i}\right)+g(z) e_{i} d y\right) e_{i} .
$$


By (4.6), $z(x)$ solves the equation

$$
\int_{\Omega}(\nabla z \mid \nabla v) d x+\int_{\Omega}|\nabla z|^{p-2}(\nabla z \mid \nabla v) d x+\int_{\Omega} g(z) v d x=\int_{\Omega} r v d x
$$

for any $v \in W_{0}^{1, p}(\Omega)$. Since $V \subset L^{\infty}(\Omega)$, we have $r(x) \in L^{\infty}(\Omega)$. Moreover $\|r\|_{\infty} \leqslant C$ where $C$ is a positive constant depending on $a$. Now as in Lemma 2.1, we can choose $v=\chi_{j}(z) \in W_{0}^{1, p}(\Omega)$ as test function in (4.7) and then we get

$$
\int_{\Omega_{j}}|\nabla z|^{p} d x \leqslant c_{1} \int_{\Omega_{j}}|z|^{q+2} d x+c_{2} \int_{\Omega_{j}}|z| d x
$$

where $\Omega_{j}=\{x \in \Omega:|z(x)|>j\}$. Applying inequality (3.4) in [13], we conclude $z \in L^{\infty}(\Omega)$ and that there exists $K>0$ such that $\|z\|_{\infty} \leqslant K$, with $K=K(a)$.

LEMmA 4.4. - Let $N \geqslant p$. For any $M>0$ there exist $r_{0}>0$ and $C>0$ such that for any $z \in W_{0}^{1, p}(\Omega) \cap L^{\infty}(\Omega)$, with $\|z\|_{\infty} \leqslant M,\|z-u\|<r_{0}$, we have

$$
\left\langle f^{\prime \prime}(z) v, v\right\rangle \geqslant C\|v\|_{b}^{2}
$$

for any $v \in W$.

Proof. - By contradiction, we assume that there exist $M>0$ and two sequences $z_{n} \in$ $W_{0}^{1, p}(\Omega) \cap L^{\infty}(\Omega)$ and $v_{n} \in W \backslash\{0\}$, such that $\left\|z_{n}\right\|_{\infty} \leqslant M,\left\|v_{n}\right\|_{W_{0}^{1,2}}=1,\left\|z_{n}-u\right\| \rightarrow 0$ and

$$
\liminf _{n \rightarrow \infty}\left\langle f^{\prime \prime}\left(z_{n}\right) v_{n}, v_{n}\right\rangle \leqslant 0 .
$$

There exists $v \in W$ such that $v_{n}$ converges to $v$ weakly in $H_{b}$ and strongly in $L^{2}(\Omega)$, up to subsequences.

Since $\left\{z_{n}\right\}$ is bounded in $L^{\infty}(\Omega)$ and $g \in C^{1}(\mathbb{R}, \mathbb{R})$, we derive

$$
\lim _{n \rightarrow \infty} \int_{\Omega} g^{\prime}\left(z_{n}\right) v_{n}^{2}=\int_{\Omega} g^{\prime}(u) v^{2} .
$$

Hence, arguing as in Lemma 4.2, by (4.9) and (4.10) we derive $v \neq 0$ and

$$
0 \geqslant \liminf _{n \rightarrow \infty}\left\langle f^{\prime \prime}\left(z_{n}\right) v_{n}, v_{n}\right\rangle \geqslant \mu\|v\|_{b}^{2},
$$

which is a contradiction.

In the next lemma we shall prove that $u$ is a strict minimum point in the direction of $W$. Precisely the following result holds.

LEMMA 4.5. - There exist $\delta>0$ such that for any $w \in W \backslash\{0\}$, with $\|w\| \leqslant \delta$, we have

$$
f(u+w)>f(u) .
$$


Proof. - In the case $N<p$ the thesis immediately follows from Lemma 4.2, with $\delta=r_{0}$. Indeed, for any $w \in W, w \neq 0$ with $\|w\|<r_{0}$ there exists $z \in W_{0}^{1, p}(\Omega)$ with $\|z-u\|<r_{0}$ such that

$$
f(u+w)-f(u)=\frac{1}{2}\left\langle f^{\prime \prime}(z) w, w\right\rangle \geqslant C\|w\|_{b}^{2}>0 .
$$

We consider the case $N \geqslant p$. By assumption $(g)$, there exist a constant $c(u)>0$, depending on the critical point $u$, and a constant $d>0$ such that for any $x \in \Omega$ and for any $s \in \mathbb{R}$ we have

$$
\left|g^{\prime}(s)\right| \leqslant c(u)+d|s-u(x)|^{p^{*}-2} .
$$

Now let us define for any $x \in \Omega$ and for any $s \in \mathbb{R}$

$$
\tilde{g}(x, s)=g(s)+\frac{d}{p^{*}-1}|s-u(x)|^{p^{*}-2}(s-u(x))
$$

and

$$
\tilde{G}(x, s)=G(s)+\frac{d}{p^{*}\left(p^{*}-1\right)}|s-u(x)|^{p^{*}} .
$$

By (4.12) it is immediate to check that

$$
D_{s} \tilde{g}(x, s) \geqslant-c(u) .
$$

As in Lemma 4.3, set $h(\xi)=\frac{1}{p}|\xi|^{p}$ for any $\xi \in \mathbb{R}^{N}$. Obviously there exist $C_{1}>0$ and $C_{2}>0$ such that $\left|h^{\prime \prime}(\xi)\right| \leqslant C_{1}|\xi|^{p-2}$ for any $\xi \in \mathbb{R}^{N}$ and $\left|h^{\prime \prime}\left(\xi_{1}-\xi_{2}\right)\right| \leqslant C_{2}\left(\left|\xi_{1}\right|^{p-2}+\right.$ $\left|\xi_{2}\right|^{p-2}$ ) for any $\xi_{1}, \xi_{2} \in \mathbb{R}^{N}$.

Now let us fix $\varepsilon>0$ such that

$$
1-C_{2} \varepsilon-C_{2} \varepsilon\|\nabla u\|_{\infty}^{p-2} \geqslant 1 / 2, \quad \mu-2 C_{2} \varepsilon\|\nabla u\|_{\infty}^{p-2} \geqslant \mu / 2 .
$$

Moreover let us define the functional $t_{\varepsilon}: W_{0}^{1, p}\left(\mathbb{R}^{N}\right) \rightarrow \mathbb{R}$ by setting

$$
t_{\varepsilon}(v)=\frac{\varepsilon}{p} \int_{\Omega}|\nabla v-\nabla u|^{p} d x-\frac{d}{p^{*}\left(p^{*}-1\right)} \int_{\Omega}|v-u|^{p^{*}} d x, \quad v \in W_{0}^{1, p}(\Omega)
$$

and let us set

$$
\tilde{f}(v)=f(v)-t_{\varepsilon}(v), \quad v \in W_{0}^{1, p}(\Omega) .
$$

Firstly, we observe that there exist $\gamma>0$ and $\varepsilon^{\prime}>0$ such that

$$
t_{\varepsilon}(v) \geqslant \varepsilon^{\prime} \int_{\Omega}|\nabla v-\nabla u|^{p} d x
$$

for any $v \in W_{0}^{1, p}(\Omega)$ such that $\|v-u\| \leqslant \gamma$. 
Now we shall prove that there exist $\sigma>0, \tilde{C}>0$ such that for any $z \in W_{0}^{1, p}(\Omega)$ with $\|z-u\| \leqslant \sigma$ we have

$$
\left\langle\tilde{f}^{\prime \prime}(z) v, v\right\rangle \geqslant \tilde{C}\|v\|_{b}^{2}
$$

for any $v \in W$. By contradiction, we assume that there exist two sequences $z_{n} \in W_{0}^{1, p}(\Omega)$ and $v_{n} \in W \backslash\{0\}$, such that $\left\|v_{n}\right\|_{W_{0}^{1,2}}=1,\left\|z_{n}-u\right\| \rightarrow 0$ and

$$
\liminf _{n \rightarrow \infty}\left\langle\tilde{f}^{\prime \prime}\left(z_{n}\right) v_{n}, v_{n}\right\rangle \leqslant 0 \text {. }
$$

There exists $v \in W$ such that $v_{n}$ converges to $v$ weakly in $H_{b}$ and strongly in $L^{2}(\Omega)$, up to subsequences. Firstly we prove that $v \neq 0$. By contradiction we assume that $v=0$. By (4.13) we have

$$
\begin{aligned}
\left\langle\tilde{f}^{\prime \prime}\left(z_{n}\right) v_{n}, v_{n}\right\rangle= & \int_{\Omega}\left|\nabla v_{n}\right|^{2} d x+\int_{\Omega}\left(h^{\prime \prime}\left(\nabla z_{n}\right) \nabla v_{n} \mid \nabla v_{n}\right) d x \\
& -\varepsilon \int_{\Omega}\left(h^{\prime \prime}\left(\nabla z_{n}-\nabla u\right) \nabla v_{n} \mid \nabla v_{n}\right) d x+\int_{\Omega} \tilde{g}^{\prime}\left(x, z_{n}\right) v_{n}^{2} d x \\
\geqslant & 1+\left(1-C_{2} \varepsilon\right) \int_{\Omega}\left|\nabla z_{n}\right|^{p-2}\left|\nabla v_{n}\right|^{2} d x \\
& -C_{2} \varepsilon\|\nabla u\|_{\infty}^{p-2} \int_{\Omega}\left|\nabla v_{n}\right|^{2} d x-c(u) \int_{\Omega} v_{n}^{2} d x \\
\geqslant & 1-\varepsilon C_{2}\|\nabla u\|_{\infty}^{p-2}-c(u) \int_{\Omega} v_{n}^{2} d x .
\end{aligned}
$$

Since $v_{n} \rightarrow 0$ in $L^{2}(\Omega)$ and (4.14) holds, we derive that (4.18) contradicts (4.17) as $n \rightarrow+\infty$ and thus $v \neq 0$.

Furthermore by (4.13), we also infer that

$$
\int_{\Omega} g^{\prime}(u) v^{2} d x \leqslant \liminf _{n \rightarrow \infty} \int_{\Omega} \tilde{g}^{\prime}\left(x, z_{n}\right) v_{n}^{2} .
$$

Applying Theorem 4.1, by (4.19) and (4.14) we have

$$
\begin{aligned}
0 \geqslant & \liminf _{n \rightarrow \infty}\left\langle\tilde{f}^{\prime \prime}\left(z_{n}\right) v_{n}, v_{n}\right\rangle \\
\geqslant & \liminf _{n \rightarrow \infty}\left(\int_{\Omega}\left(1-\varepsilon C_{2}|\nabla u|^{p-2}\right)\left|\nabla v_{n}\right|^{2} d x+\int_{\Omega}\left(1-\varepsilon C_{2}\right)\left|\nabla z_{n}\right|^{p-2}\left|\nabla v_{n}\right|^{2} d x\right. \\
& \left.+(p-2) \int_{\Omega}\left|\nabla z_{n}\right|^{p-4}\left|\left(\nabla z_{n} \mid \nabla v_{n}\right)\right|^{2} d x+\int_{\Omega} \tilde{g}^{\prime}\left(x, z_{n}\right) v_{n}^{2} d x\right) \\
\geqslant & \left(1-2 \varepsilon C_{2}\|\nabla u\|_{\infty}^{p-2}\right) \int_{\Omega}|\nabla v|^{2} d x+\int_{\Omega}\left(h^{\prime \prime}(\nabla u) \nabla v \mid \nabla v\right) d x+\int_{\Omega} g^{\prime}(u) v^{2} d x
\end{aligned}
$$




$$
=\left\langle L_{b} v, v\right\rangle-2 \varepsilon C_{2}\|\nabla u\|_{\infty}^{p-2} \int_{\Omega}|\nabla v|^{2} \geqslant \mu\|v\|_{b}^{2}-\frac{\mu}{2} \int_{\Omega}|\nabla v|^{2} \geqslant \frac{\mu}{2}\|v\|_{b}^{2}
$$

which is a contradiction.

At this point, for any $w \in W$ with $\|w\| \leqslant \min \{\gamma, \sigma\}$ we have

$$
f(u+w)-f(u)=t_{\varepsilon}(u+w)+\tilde{f}(u+w)-\tilde{f}(u) .
$$

Moreover for any $w \in W$ with $\|w\| \leqslant \min \{\gamma, \sigma\}$ there exists $z \in W_{0}^{1, p}(\Omega)$ with $\| z-$ $u \| \leqslant \min \{\gamma, \sigma\}$ such that

$$
\tilde{f}(u+w)-\tilde{f}(u)=\frac{1}{2}\left\langle\tilde{f}^{\prime \prime}(z) w, w\right\rangle
$$

and by (4.16)

$$
\tilde{f}(u+w)-\tilde{f}(u) \geqslant \tilde{C}\|w\|_{b}^{2}
$$

where $\tilde{C}$ is a suitable positive constant. Finally by (4.15), (4.20) and (4.21) we get (4.11).

Now we are ready to prove the following crucial result.

LEMMA 4.6. - There exist $r \in] 0, \delta[$ and $\rho \in] 0, r\left[\right.$ such that for any $v \in V \cap \bar{B}_{\rho}(0)$ there exists one and only one $\bar{w} \in W \cap B_{r}(0) \cap L^{\infty}(\Omega)$ such that for any $z \in W \cap \bar{B}_{r}(0)$ we have

$$
f(v+\bar{w}+u) \leqslant f(v+z+u) .
$$

Moreover $\bar{w}$ is the only element of $W \cap \bar{B}_{r}(0)$ such that

$$
\left\langle f^{\prime}(u+v+\bar{w}), z\right\rangle=0 \quad \forall z \in W .
$$

Furthermore $u$ is the only critical point of $B_{r}(u)$ and $B_{r}(u) \subset f^{c+1}$.

Proof. - We begin to consider the case $N \geqslant p$. Since $u$ is an isolated critical point of $f$ and $f$ is continuous, we can fix $0<a<\delta$ such that $u$ is the only critical point of $f$ in $B_{a}(u)$ and $B_{a}(u) \subset f^{c+1}$. By Lemma 4.3, if $z \in B_{a}(u)$ is a solution of $\left\langle f^{\prime}(z), w\right\rangle=0$ for any $w \in W$, then $z \in L^{\infty}(\Omega)$ and $\|z\|_{\infty} \leqslant M$ where $M>0$ is a positive constant, depending on $a$. Now by Lemma 4.4, in correspondence of $2 M$, there exists $\left.r_{0} \in\right] 0, a$ [ and $C>0$ such that (4.8) holds.

Now let $r \in] 0, \frac{r_{0}}{3}[$. Firstly, we notice that $f$ is sequentially lower semicontinuous with respect to the weak topology of $W_{0}^{1, p}(\Omega)$. Therefore let us fix $v \in V \cap B_{r}(0)$, there exists a minimum point $\bar{w} \in W \cap \bar{B}_{r}(0)$ of the function $w \in W \cap \bar{B}_{r}(0) \mapsto f(u+v+w)$.

We shall prove that there exists $\rho \in] 0, r\left[\right.$ such that for any $v \in V \cap \bar{B}_{\rho}(0)$ we have

$$
\inf \{f(u+v+w): w \in W,\|w\|=r\}>f(u+v) .
$$

Arguing by contradiction, we assume that there exist a sequence $\left\{w_{n}\right\}$ in $W \cap \partial B_{r}(0)$ and a sequence $\left\{v_{n}\right\}$ in $V$ with $\left\|v_{n}\right\| \rightarrow 0$ as $n \rightarrow+\infty$ such that

$$
f\left(u+v_{n}+w_{n}\right) \leqslant f\left(u+v_{n}\right) .
$$


Since $\left\{w_{n}\right\}$ is bounded, there exists $\tilde{w} \in W$ such that $w_{n}$ weakly converges to $\tilde{w}$ in $W_{0}^{1, p}(\Omega)$.

By Lemma 4.5, 0 is the unique minimum point of the function $w \in W \cap \bar{B}_{r}(0) \mapsto$ $f(u+w)$, therefore we have

$$
f(u) \leqslant f(u+\tilde{w}) .
$$

By (4.24) and (4.25), it follows that

$$
f(u)=f(u+\tilde{w})=\lim _{n \rightarrow+\infty} f\left(u+v_{n}+w_{n}\right) .
$$

In particular by (4.26)

$$
\lim _{n \rightarrow+\infty} \int_{\Omega}\left|\nabla u+\nabla v_{n}+\nabla w_{n}\right|^{p} d x=\int_{\Omega}|\nabla u+\nabla \tilde{w}|^{p} d x
$$

and thus $\left\{w_{n}\right\}$ converges to $\tilde{w}$ strongly in $W_{0}^{1, p}(\Omega)$. It follows that $\|\tilde{w}\|=r$ which contradicts (4.26).

As a consequence we infer that there exists $\rho \in] 0, r\left[\right.$ such that for any $v \in V \cap \bar{B}_{\rho}(0)$, (4.23) holds. Therefore we have that for any $v \in V \cap \bar{B}_{\rho}(0)$ the minimum point $\bar{w}$ belongs to $W \cap B_{r}(0)$ and then $\left\langle f^{\prime}(u+v+\bar{w}), z\right\rangle=0$ for any $z \in W$. Moreover by Lemma 4.3, $\bar{w} \in L^{\infty}(\Omega)$ and $\|u+v+\bar{w}\|_{\infty} \leqslant M$. Now we shall prove that for any fixed $v \in V \cap \bar{B}_{\rho}(0)$, the minimum point $\bar{w}$ is unique.

In fact, we shall prove even more, namely that $\bar{w}$ is the only element of $W \cap \bar{B}_{r}(0)$ such that

$$
\left\langle f^{\prime}(u+v+\bar{w}), z\right\rangle=0 \quad \forall z \in W .
$$

By contradiction, we suppose that there exist $w_{1}, w_{2} \in W \cap B_{r}(0) \cap L^{\infty}(\Omega), w_{1} \neq w_{2}$ such that $\left\langle f^{\prime}\left(u+v+w_{1}\right), z\right\rangle=0$ and $\left\langle f^{\prime}\left(u+v+w_{2}\right), z\right\rangle=0$ for any $z \in W$.

We notice that $\left\|v+w_{1}+t\left(w_{2}-w_{1}\right)\right\|=\left\|v+w_{1}(1-t)+w_{2}\right\| \leqslant 3 r$ for any $t \in[0,1]$ and then $\left\|v+w_{1}+t\left(w_{2}-w_{1}\right)\right\| \leqslant 3 r<r_{0}$ for any $t \in[0,1]$. Furthermore we have $\left\|u+v+w_{1}+t\left(w_{2}-w_{1}\right)\right\|_{\infty} \leqslant 2 M$. Therefore, by (4.8), we deduce

$$
\begin{aligned}
0 & =\left\langle f^{\prime}\left(u+v+w_{1}\right)-f^{\prime}\left(u+v+w_{2}\right), w_{1}-w_{2}\right\rangle \\
& =\int_{0}^{1}\left\langle f^{\prime \prime}\left(u+v+w_{1}+t\left(w_{2}-w_{1}\right)\right)\left(w_{1}-w_{2}\right), w_{1}-w_{2}\right\rangle d t>0 .
\end{aligned}
$$

The claim is proved.

In the case $N<p$ the proof is easier and the thesis immediately follows by Lemma 4.2 , arguing as before.

\section{Proof of the main result}

We can define the map $\psi: v \in V \cap \bar{B}_{\rho}(0) \mapsto \bar{w} \in W \cap B_{r}(0)$ where $\bar{w}$ is the unique minimum point of the function $w \in W \cap \bar{B}_{r}(0) \mapsto f(u+v+w)$. 
By Lemma 4.6, for any $v \in V \cap \bar{B}_{\rho}(0)$ and $w \in W \cap B_{r}(0), w=\psi(v)$ if and only if $\left\langle f^{\prime}(u+v+w), z\right\rangle=0$ for any $z \in W$.

Now we shall prove that $\psi$ is a continuous map. Let $\left\{v_{n}\right\}$ be a sequence in $V \cap \bar{B}_{\rho}(0)$ such that $v_{n}$ converges strongly to $v \in V$ as $n \rightarrow+\infty$. Since $\psi\left(v_{n}\right)$ is bounded in $W_{0}^{1, p}(\Omega)$, then $\psi\left(v_{n}\right)$ weakly converges to $w \in W$. Let us prove that $w=\psi(v)$. Firstly we notice that

$$
f\left(u+v_{n}+\psi\left(v_{n}\right)\right) \leqslant f\left(u+v_{n}+z\right)
$$

for any $n \in \mathbb{N}$ and $z \in W \cap \bar{B}_{r}(0)$. Therefore, by (5.1), we have

$$
\begin{aligned}
f(u+v+w) & \leqslant \liminf _{n \rightarrow+\infty} f\left(u+v_{n}+\psi\left(v_{n}\right)\right) \\
& \leqslant \limsup _{n \rightarrow+\infty} f\left(u+v_{n}+z\right)=f(u+v+z)
\end{aligned}
$$

for any $z \in W \cap \bar{B}_{r}(0)$. As a consequence we deduce $w=\psi(v)$. Moreover by (5.2) we deduce

$$
\lim _{n \rightarrow+\infty} f\left(u+v_{n}+\psi\left(v_{n}\right)\right)=f(u+v+\psi(v))
$$

and so $\left\|\psi\left(v_{n}\right)\right\| \rightarrow\|\psi(v)\|$, as $n \rightarrow+\infty$, which proves that $\psi$ is a continuous map.

At this point we define the map $\phi: V \cap \bar{B}_{\rho}(0) \rightarrow \mathbb{R}$ setting $\phi(v)=f(u+v+\psi(v))$; $\phi$ is a continuous map. Furthermore let us set

$$
Y=\left\{u+v+\psi(v): v \in V \cap \bar{B}_{\rho}(0)\right\} .
$$

Since $\psi$ is a continuous map and $\psi(0)=0$, it follows that the pair $\left(\phi^{c}, \phi^{c} \backslash\{0\}\right)$ is homeomorphic to $\left(\left(f_{\mid Y}\right)^{c},\left(f_{\mid Y}\right)^{c} \backslash\{u\}\right)$, where

$$
\phi^{c}=\left\{v \in V \cap \bar{B}_{\rho}(0): f(u+v+\psi(v)) \leqslant c\right\}
$$

and

$$
\left(f_{\mid Y}\right)^{c}=\left\{u+v+\psi(v): v \in V \cap \bar{B}_{\rho}(0), f(u+v+\psi(v)) \leqslant c\right\} .
$$

Therefore we have

$$
C_{j}(\phi, 0)=C_{j}\left(f_{\mid Y}, u\right) .
$$

Now our aim is to show that

$$
C_{j}(f, u)=C_{j}\left(f_{\mid Y}, u\right)
$$

since this permits to complete the proof of Theorems 1.1 and 1.2 as follows.

Proof of Theorem 1.1. - First of all Lemma 3.2 and Eq. (3.2) assure that $m(f, u)$ is finite. Moreover as $u$ is a local isolated maximum of $f$ along the finite dimensional space $V=H^{-}$, we deduce, by construction, that 0 is a local maximum of $\phi$ in $V$. Therefore $C_{j}(\phi, 0) \cong \mathbb{K}$, if $j=\operatorname{dim} V=m(f, u)$ and $C_{j}(\phi, 0)=\{0\}$ for any $j \neq m(f, u)$. Finally, by (5.4), (5.5) we deduce $C_{j}(f, u)=C_{j}(\phi, 0)$ and the thesis follows. 
Proof of Theorem 1.2. - Lemma 3.2 and Eq. (3.2) assure that $m(f, u)$ and $m^{*}(f, u)$ are finite. From Theorem 2.6, due to Lancelotti [14], we have that $C_{q}(f, u)=\{0\}$ for any $q \leqslant m(f, u)-1$. On the other hand, we infer $C_{j}(\phi, 0)=\{0\}$ for any $j>\operatorname{dim} V=$ $m^{*}(f, u)$. By (5.4), (5.5) we deduce $C_{j}(f, u)=C_{j}(\phi, 0)$ and the thesis follows.

In order to prove relation (5.5) it is useful to distinguish again between the two cases $N<p$ and $N \geqslant p$, treating them separately in the two following subsections.

\subsection{Case $N<p$}

For convenience, let us set

$$
U=u+\left(V \cap \bar{B}_{\rho}(0)\right)+\left(W \cap \bar{B}_{r}(0)\right) .
$$

We shall prove that

$$
\left(\left(f_{\mid Y}\right)^{c},\left(f_{\mid Y}\right)^{c} \backslash\{u\}\right)
$$

is a deformation retract of

$$
\left(f^{c} \cap U, f^{c} \cap U \backslash\{u\}\right) .
$$

Set $A=f^{c} \cap U$ and $B=f^{c} \cap U \backslash\{u\}$, let us define

$$
\gamma(t, u+v+w)=u+v+w+t(\psi(v)-w)
$$

where $t \in[0,1]$ and $x=u+v+w \in A$. By $(4.1), f(\gamma(t, x)) \leqslant c$ for any $t \in[0,1]$ and $x \in A$, so that $\gamma:[0,1] \times A \rightarrow A$ is well posed. Moreover it is easy to see that $\gamma$ is continuous, $\gamma(0, \cdot)=i d_{A}, \gamma(1, A) \subset\left(f_{\mid Y}\right)^{c}$ and for any $t \in[0,1], \gamma(t, \cdot)_{\mid\left(f_{\mid Y}\right)^{c}}=i d_{\left(f_{\mid Y}\right)^{c}}$. Furthermore, since 0 is the minimum point of the function $w \mapsto f(u+w)$, we can deduce that for any $t \in[0,1], \gamma(t, B) \subset B$, and that $\gamma(1, B) \subset\left(f_{\mid Y}\right)^{c} \backslash\{u\}$.

Thus we can conclude that

$$
H^{j}\left(f_{\mid Y}^{c}, f_{\mid Y}^{c} \backslash\{u\}\right)
$$

is isomorphic to

$$
H^{j}\left(f^{c} \cap U, f^{c} \cap U \backslash\{u\}\right)
$$

and by the definition of critical groups and Remark 2.2 we derive (5.5).

\subsection{Case $N \geqslant p$}

In this case (4.1) is not verified, so that, following the notations of the previous subsection, it can happen that $f(\gamma(t, x))>c$ for some $(t, x) \in] 0,1\left[\times\left(f^{c} \cap U\right)\right.$.

In order to overcome this difficulty, we basically follow the ideas of Second Deformation Lemma (cf. [5,6]), modifying them for our use. We report here the main passages for the convenience of the reader.

First of all, for each $z \in U$ let us define

$$
\alpha(z)=\sup _{w \in W \backslash\{0\}} \frac{\left\langle f^{\prime}(z), w\right\rangle}{\|w\|} .
$$


From continuity of $f^{\prime}$ we get that $\alpha: U \rightarrow \mathbb{R}$ is continuous too. Moreover we know from Lemma 4.6 that, for each $z \in U, \alpha(z)=0 \Leftrightarrow z \in Y$.

By standard arguments, concerning the construction of a pseudogradient vector field, we can obtain the following lemma (see e.g., [15]).

LEMma 5.1. - Set $U^{*}=U \backslash Y$, there exists a locally Lipschitz continuous vector field $X: U^{*} \rightarrow W$ such that, for each $z \in U^{*}$

(1) $\|X(z)\| \leqslant 2 \alpha(z)$,

(2) $\left\langle f^{\prime}(z), X(z)\right\rangle \geqslant \alpha^{2}(z)$.

The existence of the vector field $X$ gives a decreasing flow for $f$ which is in the direction of $W$. In fact the following Cauchy problem

$$
\left\{\begin{array}{l}
\dot{\sigma}(t)=-X(\sigma(t)) \\
\sigma(0)=z_{0} \in U^{*}
\end{array}\right.
$$

is locally solvable, and the function $t \mapsto f(\sigma(t))$ is decreasing, as

$$
\frac{d}{d t} f(\sigma(t))=\left\langle f^{\prime}(\sigma(t)), \dot{\sigma}(t)\right\rangle=-\left\langle f^{\prime}(\sigma(t)), X(\sigma(t))\right\rangle<-\alpha^{2}(\sigma(t)) .
$$

Next lemma states a sort of (P.S.) condition for $f$ in the direction of $W$.

LEMMA 5.2. - For any sequence $\left\{z_{n}\right\} \subset U$ such that $\alpha\left(z_{n}\right) \rightarrow 0$, as $n \rightarrow+\infty$, there is a convergent subsequence.

Proof. - Let $\left\{e_{1}, \ldots, e_{m^{*}}\right\}$ be an $L^{2}$-orthonormal basis in $V$, where $m^{*}=m^{*}(f, u)$, and let $P_{W}: W_{0}^{1, p}(\Omega) \rightarrow W$ be the projection on $W$. For any $z \in W_{0}^{1, p}(\Omega)$

$$
\left\langle f^{\prime}\left(z_{n}\right), z\right\rangle=\left\langle f^{\prime}\left(z_{n}\right), P_{W}(z)\right\rangle+\sum_{i=1}^{m^{*}} \int_{\Omega} \alpha_{n}^{i} e_{i} z d x,
$$

where $\alpha_{n}^{i}=\left\langle f^{\prime}\left(z_{n}\right), e_{i}\right\rangle$. As $\left\{z_{n}\right\}$ is bounded and $\alpha\left(z_{n}\right) \rightarrow 0$, we have that $f^{\prime}\left(z_{n}\right)$ converges to $h: W_{0}^{1, p}(\Omega) \rightarrow \mathbb{R}$, defined by $h(z)=\sum_{i=1}^{m^{*}} \alpha_{i} \int_{\Omega} e_{i} z d x$ for any $z \in W_{0}^{1, p}(\Omega)$, where $\alpha_{n}^{i} \rightarrow \alpha_{i} \in \mathbb{R}$. Moreover we notice that $f^{\prime}=A+B$ where $A, B: W_{0}^{1, p}(\Omega) \rightarrow$ $W^{-1, p^{\prime}}(\Omega)$ are defined as follows

$$
\begin{gathered}
\langle A(z), \zeta\rangle=\int_{\Omega}\left(|\nabla z|^{p-2}(\nabla z \mid \nabla \zeta)+(\nabla z \mid \nabla \zeta)\right) d x, \\
\langle B(z), \zeta\rangle=\int_{\Omega} g(z) \zeta d x, \quad \forall z, \zeta \in W_{0}^{1, p}(\Omega) .
\end{gathered}
$$

As $A$ is an invertible map with continuous inverse (cf. Theorem 5 in [2]) and $B$ is compact, we obtain the convergence up to subsequences of $\left\{z_{n}\right\}$.

COROLlaRY 5.3. - If $\bar{A} \subset U^{*}$, then there exists $\varepsilon>0$ such that $\inf _{z \in A} \alpha(z)>\varepsilon$ where $\bar{A}$ is the closure of $A$. 
Proof. - By contradiction, let $A$ be a set such that $\bar{A} \subset U^{*}$ and $\left\{z_{n}\right\} \subset A$ a sequence such that $\alpha\left(z_{n}\right) \rightarrow 0$, as $n \rightarrow+\infty$. By Lemma $5.2 z_{n} \rightarrow z \in \bar{A} \subset U^{*}$, while, by continuity of $\alpha, \alpha(z)=0$, which contradicts the definition of $U^{*}$.

Now let us define $\gamma:[0,1] \times\left(f^{c} \cap U\right) \rightarrow U$ so that $\gamma(t, u+v+w)=u+v+$ $(1-t) w+t \psi(v)$. The following theorem will be proved.

THEOREM 5.4. - There exists $\left.\rho_{1} \in\right] 0, \rho$ [ such that introducing the following sets

$$
\begin{aligned}
U_{1} & =u+\left(V \cap \bar{B}_{\rho_{1}}(0)\right)+\left(W \cap \bar{B}_{r}(0)\right), \\
M & =\gamma\left([0,1] \times\left(f^{c} \cap U\right)\right), \\
M_{1} & =\gamma\left([0,1] \times\left(f^{c} \cap U_{1}\right)\right),
\end{aligned}
$$

we have $f^{c} \cap U_{1}$ is a retract of $M_{1}$ in the direction of $W$. Namely, there exists a continuous map $r: M_{1} \rightarrow f^{c} \cap U_{1}$ such that $\left.r\right|_{f^{c} \cap U_{1}}=i d_{f^{c} \cap U_{1}}$ and

$$
\forall z \in M_{1}: r(z)-z \in W .
$$

Proof. - First of all we will show that there is $\left.\rho_{1} \in\right] 0, \rho[$ such that

$$
f(u+v+w)<f(u+v+z)
$$

for each $u+v+w \in M$ with $v \in V \cap \bar{B}_{\rho_{1}}(0)$, and for each $z \in W$ with $\|z\|=r$. Indeed, reasoning by contradiction, we assume that there exist three sequences $\left\{v_{n}\right\} \subset V$, $\left\{w_{n}\right\} \subset W$ and $\left\{z_{n}\right\} \subset W$ such that $v_{n} \rightarrow 0, u+v_{n}+w_{n} \in M,\left\|z_{n}\right\|=r$ and

$$
f\left(u+v_{n}+z_{n}\right) \leqslant f\left(u+v_{n}+w_{n}\right) \quad \forall n \in \mathbb{N} .
$$

First notice that the definition of $M$ and the fact that 0 is the minimum point for the function $w \mapsto f(u+w)$ give that $w_{n} \rightarrow 0$, as $n \rightarrow+\infty$.

As $\left\{z_{n}\right\}$ is bounded, it converges weakly to a certain $z \in W$ and by (5.9) we have $f(u+z)=f(u)$, thus $z=0$. Moreover by (5.9) we can also infer that $\left\|z_{n}\right\|$ converges to $\|z\|$ and $\|z\|=r$, in contradiction with $z=0$.

We recall that $M_{1} \subset f^{c+1}$, as $M_{1} \subset U_{1} \subset B_{r}(u)$ and last assertion of Lemma 4.6 assures $B_{r}(u) \subset f^{c+1}$.

Now let $z_{0}$ be an element of $M_{1} \cap f^{-1}[c, c+1] \backslash Y$. This means that $z_{0}=u+v_{0}+w_{0}$, $w_{0} \neq \psi\left(v_{0}\right)$ and $f\left(u+v_{0}+\psi\left(v_{0}\right)\right)<c$. Thus the Cauchy problem

$$
\left\{\begin{array}{l}
\dot{\sigma}(t)=-X(\sigma(t)) \\
\sigma(0)=z_{0}
\end{array}\right.
$$

is locally solvable.

Thus, reasoning as in Second Deformation Theorem, $\exists T_{z_{0}} \geqslant 0$ such that solution $\sigma\left(t, z_{0}\right)$ to $\left(P_{z_{0}}\right)$ is defined at least in $\left[0, T_{z_{0}}\right]$ and $f\left(\sigma\left(T_{z_{0}}, z_{0}\right)\right)=c$ (notice that $T_{z_{0}}=0$, if $\left.f\left(z_{0}\right)=c\right)$.

We point out that relations (5.6) and (5.8) assure that $\sigma\left(T_{z_{0}}, z_{0}\right) \in U_{1}$.

Moreover the function $z \mapsto T_{z}$ is continuous. Indeed, let $z_{0}$ be an element of $M_{1} \cap f^{-1}[c, c+1] \backslash Y$, so that $z_{0}=u+v_{0}+w_{0}$ and $f\left(u+v_{0}+\psi\left(v_{0}\right)\right)<c$. By 
continuity of $f$ and $\psi$, there exists a closed neighborhood $U_{z_{0}}$ of $z_{0}$ such that, writing $z=u+v+w$, we have that $f(u+v+\psi(v))<c$ for each $z \in U_{z_{0}}$.

Hence the set $A=\left\{\sigma(t, z): z \in U_{z_{0}}, t \in\left[0, T_{z_{0}}\right]\right\}$ is a closed subset of $U^{*}$ and, by Corollary 5.3, there exists $\varepsilon_{0}>0$ such that $\alpha(z)>\varepsilon_{0}$ for each $z \in A$. Now $t=T_{z}$ is the solution of $f(\sigma(t, z))=c$, where by $(5.6)$,

$$
\frac{\delta}{\delta t} f(\sigma(t, z))<-\alpha^{2}(\sigma(t, z))<-\varepsilon_{0}^{2},
$$

so continuity of $z \mapsto T_{z}$ is assured by implicit function theorem.

Now we are ready to define the function $r: M_{1} \rightarrow f^{c} \cap U_{1}$ as follows

$$
r(z)= \begin{cases}z & \text { if } z \in f^{c} \\ \sigma\left(T_{z}, z\right) & \text { elsewhere }\end{cases}
$$

Relation (5.7) comes from the definition of $X$ given in Lemma 5.1.

Moreover it is clear that $r$ is continuous in the interior of $f^{c} \cap U_{1}$, while continuity in $M_{1} \backslash f^{c}$ and in $f^{-1}\{c\} \backslash Y$ derives from ODE theory, thus it remains only to verify continuity of $r$ in $f^{-1}\{c\} \cap Y$.

So let us fix $z_{0}$ in $f^{-1}\{c\} \cap Y$, so that $z_{0}=u+v_{0}+\psi\left(v_{0}\right)$, and let $\left\{z_{n}\right\}_{n \in \mathbb{N}}$ be a sequence such that $z_{n} \rightarrow z_{0}$. If $z_{n}$ eventually belongs to $f^{c}$, then the assert easily comes, so let us suppose $z_{n} \notin f^{c}$ for each $n$. This means that $z_{n}=u+v_{n}+w_{n}$ where $v_{n} \rightarrow v_{0}$ by continuity of $P_{V}$, and $r\left(z_{n}\right)=u+v_{n}+\zeta_{n}$ where $f\left(u+v_{n}+\zeta_{n}\right)=c$ and $\left\{\zeta_{n}\right\} \subset W \cap B_{r}(0)$.

Thus, denoting by $\zeta$ the weak limit of $\zeta_{n}$, we have that

$$
f\left(u+v_{0}+\zeta\right) \leqslant \lim _{n \rightarrow+\infty} f\left(u+v_{n}+\zeta_{n}\right)=c=f\left(u+v_{0}+\psi\left(v_{0}\right)\right) .
$$

Consequently $\zeta=\psi\left(v_{0}\right)$ and $\zeta_{n} \rightarrow \psi\left(v_{0}\right)$ strongly.

So $r\left(z_{n}\right) \rightarrow z_{0}=r\left(z_{0}\right)$ and the assert is completely proved.

In order to prove relation (5.5), which is the aim of this subsection, it is sufficient to show that

$$
\left(\left(\left.f\right|_{Y}\right)^{c} \cap U_{1},\left(\left.f\right|_{Y}\right)^{c} \cap U_{1} \backslash\{u\}\right)
$$

is a deformation retract of

$$
\left(f^{c} \cap U_{1}, f^{c} \cap U_{1} \backslash\{u\}\right)
$$

and the previous theorem makes possible to obtain the desired homotopy equivalence between the two pairs.

We write the previous sets as

$$
\begin{aligned}
A=f^{c} \cap U_{1}, & A^{\prime} & =f^{c} \cap U_{1} \backslash\{u\}, \\
B=\left(\left.f\right|_{Y}\right)^{c} \cap U_{1}, & B^{\prime} & =\left(\left.f\right|_{Y}\right)^{c} \cap U_{1} \backslash\{u\} .
\end{aligned}
$$

Now let us define

$$
\eta(t, u+v+w)=r(u+v+w+t(\psi(v)-w))
$$


where $t \in[0,1]$ and $x=u+v+w \in A$. By Theorem 5.4, $f(\eta(t, x)) \leqslant c$ for any $t \in[0,1]$ and $x \in A$, so that $\eta:[0,1] \times A \rightarrow A$ is well posed. Moreover it is easy to see that $\eta$ is continuous, $\eta(0, \cdot)=i d_{A}, \eta(1, A) \subset A^{\prime}$ and for any $t \in[0,1], \eta(t, \cdot)_{A^{\prime}}=i d_{A^{\prime}}$. Furthermore by Lemma 4.5 and relation (5.7) we can deduce that $\eta(1, B) \subset B^{\prime}$ and that $\eta(t, B) \subset B$ for any $t \in[0,1]$.

Thus $\left(A^{\prime}, B^{\prime}\right)$ is proved to be a deformation retract of $(A, B)$ and, by Remark 2.2, relation (5.5) comes.

\section{Acknowledgements}

We wish to express special thanks to Prof. Vieri Benci for having suggested us the topic and to Prof. Marco Degiovanni for stimulating discussions and helpful suggestions.

\section{REFERENCES}

[1] D. Arcoya, L. Boccardo, Critical points for multiple integrands of the calculus of variations, Arch. Rat. Mech. Anal. 134 (1996) 249-274.

[2] V. Benci, P. D’Avenia, D. Fortunato, L. Pisani, Solitons in several space dimensions: a Derrick's problem and infinitely many solutions, Arch. Rat. Mech. Anal. 154 (2000) 297-324.

[3] V. Benci, D. Fortunato, L. Pisani, Soliton-like solutions of a Lorentz invariant equation in dimension 3, Math. Phys. 3 (1998) 315-344.

[4] K. Chang, Morse theory on Banach space and its applications to partial differential equations, Chin. Ann. of Math. 4B (1983) 381-399.

[5] K. Chang, Infinite Dimensional Morse Theory and Multiple Solution Problems, Birkhäuser, Boston, 1993.

[6] K. Chang, Morse theory in nonlinear analysis, in: A. Ambrosetti, K.C. Chang, I. Ekeland (Eds.), Nonlinear Functional Analysis and Applications to Differential Equations, Word Scientific, Singapore, 1998.

[7] S. Cingolani, G. Vannella, Some results on critical groups for a class of functionals defined on Sobolev Banach spaces, Rend. Acc. Naz. Lincei 12 (2001) 1-5.

[8] J.N. Corvellec, M. Degiovanni, Nontrivial solutions of quasilinear equations via nonsmooth Morse theory, J. Differential Equations 136 (1997) 268-293.

[9] E. Dibenedetto, $C^{1+\alpha}$ local regularity of weak solutions of degenerate elliptic equations, Nonlinear Analysis TMA 7 (1983) 827-850.

[10] H. Egnell, Existence an nonexistence results for $m$-Laplace equations involving critical Sobolev exponents, Arch. Rat. Mech. Anal. 104 (1988) 57-77.

[11] D. Gilbarg, N.S. Trudinger, Elliptic Partial Differential Equations of Second Order, Springer-Verlag, Berlin, 1998.

[12] A.D. Ioffe, On lower semicontinuity of integral functionals I and II, SIAM J. Control Optim. 15 (1977) 521-538, and 991-1000.

[13] O.A. Ladyzhenskaya, N.N. Ural'tseva, Linear and Quasilinear Elliptic Equations, Academic Press, New York, 1968.

[14] S. Lancelotti, Morse index estimates for continuous functionals associated with quasilinear elliptic equations, Adv. Differential Equations 7 (2002) 99-128.

[15] J. Mawhin, M. Willem, Critical Point Theory and Hamiltonian Systems, in: Applied Mathematical Sciences, Vol. 74, Springer-Verlag, New York, 1989. 
[16] F. Mercuri, G. Palmieri, Problems in extending Morse theory to Banach spaces, Boll. UMI 12 (1975) 397-401.

[17] R. Palais, Morse theory on Hilbert manifolds, Topology 2 (1963) 299-340.

[18] S. Smale, Morse theory and a non-linear generalization of the Dirichlet problem, Ann. Math. 80 (1964) 382-396.

[19] E.H. Spanier, Algebraic Topology, McGraw-Hill, New York, 1966.

[20] P. Tolksdorf, Regularity for a more general class of quasilinear elliptic equations, J. Differential Equations 51 (1984) 126-150.

[21] P. Tolksdorf, On the Dirichlet problem for a quasilinear equations in domains with conical boundary points, Comm. Part. Differential Equations 8 (1983) 773-817.

[22] A.J. Tromba, A general approach to Morse theory, J. Differential Geom. 12 (1977) 47-85.

[23] K. Uhlenbeck, Morse theory on Banach manifolds, J. Funct. Anal. 10 (1972) 430-445. 